\title{
The Precision of Different Types of Plates Fabricated with a Computer-Aided Design and Manufacturing System in Mandibular Reconstruction with Fibular Free Flaps
}

Nutcha Yodrabum ( $\nabla$ n.yodrabum@gmail.com )

Siriraj Hospital

Krit Rudeejaroonrung

Meticuly Co. Ltd, Chulalongkorn University

Natthaphat Viriya

Meticuly Co. Ltd, Chulalongkorn University

Irin Chaikangwan

Siriraj Hospital

Natthapong Kongkunnavat

Siriraj Hospital

Jirapat Tianrungroj

Siriraj Hospital

Mathee Ongsiriporn

Siriraj Hospital

Parkpoom Piyaman

Siriraj Hospital

Chedtha Puncreobutr

Chulalongkorn University

\section{Research Article}

Keywords: Computer-assisted surgery, Mandibular reconstruction, Fibular free flap

Posted Date: January 24th, 2022

DOI: https://doi.org/10.21203/rs.3.rs-1253101/v1

License: (c) (1) This work is licensed under a Creative Commons Attribution 4.0 International License. Read Full License 


\section{Abstract \\ Background}

Computer-assisted surgery (CAS) has been introduced to mandible reconstruction with fibular free flap in cutting guide placement. When CAS is cooperated with different plate fixations, the results show various degrees of errors by which this study aimed to evaluate.

\section{Methods}

Mock surgeries were conducted in 3D-printed mandibles with either 2 types of defects; limited or extensive, reconstructed from 2 ameloblastoma patients. Three types of fixations; miniplate, manually bending reconstruction plate and patient-specific plates are tested, each of which was performed 3 times in each type of defects, adding up to 18 surgeries. One with the least errors was selected and applied with patients whose 3D-printed mandibles derived. Finally, in vivo errors were compared with the mock.

\section{Results}

In limited defect, average errors show no statistical significance among all types. In extensive defect, patient-specific plate had a significantly lower average condylar error than manually bending reconstruction plate and miniplate $(8.09 \pm 2.52 \mathrm{~mm}$ vs. $25.49 \pm 2.72$ and $23.13 \pm 13.54 \mathrm{~mm}$, respectively). When patient-specific plate was applied in vivo, the errors were not significantly different from the mock.

\section{Conclusion}

Patient-specific plates cooperated with CAS shows the least errors. Nevertheless, manually bent reconstruction plates and miniplates could be applied in limited defects with caution.

\section{Introduction}

Computer-assisted surgery (CAS) has been used for head and neck surgery, including fibular free flap surgery, since the $1990 \mathrm{~s}^{1-3}$ and is widely used ${ }^{4}$. In practice, CAS consists of two components: computeraided design (CAD) and computer-assisted manufacturing (CAM) ${ }^{5,6}$. CAD/CAM and the cutting guide increase the accuracy of fibular free flap surgery ${ }^{7-9}$, decrease the operative time ${ }^{4,8}$, decrease ischaemic time $^{7}$ and are easy to use ${ }^{8}$. Furthermore, the increase in accuracy contributes directly to the aesthetic results and functional outcomes related to the position of the mandibular condyle and temporomandibular joint (TMJ) ${ }^{10,11}$.

The cutting guide transfers a mock-up to the surgical field. If the cutting guide is accurately positioned on the patient, the accuracy of other aspects of the guide will also be achieved. 
A recent study by Yang and colleagues found that the use of CAS combined with a patient-specific plate, specifically a 3D-printed customized mandibular reconstruction plate, in non-extensive defects increases the spatial accuracy of the TMJ reconstruction, yielding better results than commercially available

manually bending reconstruction plate ${ }^{12}$. Even though the patient-specific plate can provide good results, surgeons may adopt different methods for bone fixation processes such as miniplate and manually bending reconstruction plate. This indicates that most surgeons can access CAD/CAM for surgical planning and cutting guides preparation. Nevertheless, it is challenging to obtain patient-specific plate. Currently, there is no research on the precision of using cutting guides with different fixation methods.

Therefore, we investigated the errors of different fixation methods and defect extent in fibular free flap surgery using CAD/CAM and cutting guides. The three objectives of this study were to (1) compare the errors in the mandibular condyle and the fibular model among different types of fixation, (2) compare the error associated with the defect extent and type of fixation, and (3) evaluate the underlying causes of the aforementioned errors.

\section{Materials And Methods}

This study was approved by the Institutional Review Board (IRB) of the Faculty of Medicine Siriraj Hospital (Approval no. 369/2563). All methods were performed in accordance with the relevant guidelines and regulations including the Declaration of Helsinki. The informed consent was obtained from all subjects and/or their legal guardian(s). The prototypes of the mandible models were retrieved from 2 patients with ameloblastoma who had different defect characteristics. The "limit defect" involved the parasymphysis of mandible to the ipsilateral angle of mandible and required reconstruction with 2 segments of the fibula. The "extensive defect" involved the body of the mandible to the contralateral angle of mandible and required 3 fibular segments for reconstruction (Figure 1).

This study was conducted in 2 parts: one was surgical simulation in bone models, and another was mandible reconstruction with patient-specific plate in vivo. In the first part, the models were divided into 2 groups according to the extent of the defect. Bone fixation was performed with 3 types of plate: miniplate, manually bending reconstruction plate, and patient-specific plate. All model experiments were done with each type of plate three times, and the results were averaged (Figure 2). In the second part, we studied the accuracy of actual fixation with patient-specific plate in vivo in 2 patients who were used as the reference for the bone model surgery.

\section{Mandibular and Fibular Model Preparation}

Two ameloblastoma patients underwent computed tomography (CT) scans on a GE revolution system (GE Healthcare, Chicago, IL, USA), with a $0.625 \mathrm{~mm}$ thickness for the facial bone and $1.25 \mathrm{~mm}$ thickness for the lower extremity, with intravenously administered iodinated contrast medium (Iopamiro370, Bracco, Israel). Subsequently, the data, in the form of a Digital Imaging and Communications in Medicine (DICOM) file, was transformed into 3D models of the fibula and the mandible in the form of a 
stereolithography interface (STL) file using Avizo software (Thermo Fisher Scientific Inc, Waltham, MA, USA).

\section{Virtual Surgical Planning and Surgical Guide Model Preparation}

The surgeon determined the cutting position on the mandibular model. Then, the engineering team designed cutting guides for the mandible and fibula and patient-specific plate with Ansys Space Claim software (Ansys Inc, Canonsburg, PA, USA). The screw drilling positions for patient-specific plate were also indicated preoperatively.

\section{Bone Model and Surgical Guide Manufacturing}

Each set of synthetic bone models consisted of a mandible and fibula. Eighteen sets of models were used in this study. These models were manufactured with fused deposition modelling technology using biocompatible thermoplastic material. The mandibular guides and fibular guides were manufactured with the same process.

\section{Plate Preparation Method}

We prepared 3 different types of fixation systems: a $2.0 \mathrm{~mm}$ titanium miniplate (Leibinger Universal CMF Plating System, Stryker, MI, USA), a $2.3 \mathrm{~mm}$ titanium manually bending reconstruction plate (KLS Martin, Tuttlingen, Germany) and patient-specific plate (Meticuly Co Ltd, Bangkok, Thailand). The manually bending reconstruction plate was prebent with a printed reconstructed mandibular model. The patientspecific plate was manufactured with selective melting technology (Mlab cusing 200R, GE Additive, Lichtenfels, Germany) with medical-grade Ti-6Al-4 V alloy (Rematitan® CL, GE Additive, Lichtenfels, Germany).

\section{Surgical Simulation in the Bone Model}

The simulation of mandibular reconstruction with a fibular free flap was carried out using bone models. All surgical simulations were performed by the senior author (NY). First, the mandibular and fibular cutting guide was applied to the bone model. Then predrilling and screwing were implemented to define the screw positioning for the cutting guide and plate fixation. After the cutting guide was attached by screws, the bone model was cut with an electrical bone saw using the 1-mm thickness saw blade (Primado2, NSK, Kanuma, Tochigi, Japan). The metal sleeve was placed to protect the cutting guide although it caused an error. After the mandibular defect was created and the artificial fibula parts were made, each part of fibula was attached to the defect using different types of plate according to the protocol. Then, all of the simulation models and fixation parts underwent CT scanning.

\section{Mandibular Reconstruction in vivo}

The two aforementioned ameloblastoma patients underwent mandibular reconstruction after tumour eradication with fibular free flaps using CAD/CAM cutting guides and patient-specific plate. The operative steps were similar to those used in the surgical simulation of bone models. All fibular free flaps were 
viable, and no post-operative complications occurred. After 1 year of surgery, both patients underwent follow-up CT scans, the fibular free flaps were properly healed and no late complications were observed.

\section{Superimposition of Reconstructed Mandible Model}

We converted the postoperative CT scan images from the DICOM format to the STL format. Each part of the reconstructed model was independently segmented and then superimposed on the preoperative mandible. The superimposition involved a semiautomatic registration processes using two algorithms for surface alignment and few manual adjustments.

We set the large side of the remaining mandible, which was the left side for both types of defects in this study, as the reference for superimposition. To ensure the correct positioning of the superimposition, the five main reference points had to have an error less than $1 \mathrm{~mm}$ (Figure 3).

\section{Measurement, Analysis of the Results and Statistical Analysis}

The errors between the pre- and postoperative surgical simulations in bone models are presented as the mean and standard deviation. The errors among the types of plate and the extent of defect were also compared. Finally, we compared the error between surgical simulation in bone model and actual mandible reconstruction in vivo.

We also analysed the cause of error using numerical data and analysed 3D model images to determine the position of the screw hole at the surface of bone model and the direction of the screw tract within the bone model. This measurement was rely on the hypothesis that the screw position and tract reflect the precision of the cutting guide position and the outcomes of the surgery.

Data analyses were conducted using IBM SPSS Statistics, version 22.0 (IBM Corp., Armonk, NY, USA). We tested the normality of the data using the Shapiro-Wilk test. If the data had a normal distribution, the accuracy was determined by the independent $t$ test or analysis of variance (ANOVA). Kruskal-Wallis testing or independent-samples Mann-Whitney U testing was used for nonnormally distributed data. A $p$ value less than 0.05 was considered statistically significant.

\section{Results}

The experiment was conducted in eighteen bone models and two actual patients whose data had been used to construct the bone models.

\section{Effect of Plate Type for a Limit Defect and Extensive Defect}

The errors from the three types of plate are shown in figure 4. For the limit defect, both the average fibular and average condylar errors were not significantly different among plate types. The average condylar 
errors of patient-specific plate $(7.53 \pm 2.75 \mathrm{~mm})$ and manually bending reconstruction plate $(5.51 \pm 1.59$ $\mathrm{mm}$ ) were lower than those of the miniplate $(17.64 \pm 10.29 \mathrm{~mm})$ for the limit defect.

For the extensive defect, patient-specific plate had the lowest error for the condylar position $(7.50 \pm 2.48$ $\mathrm{mm})$, being significantly lower than those of manually bending reconstruction plate $(25.49 \pm 2.72 \mathrm{~mm})$ and miniplate $(23.13 \pm 13.54 \mathrm{~mm})$. There were no significant differences in error between manually bending reconstruction plate and miniplate. Regarding fibular errors, it was found that the group that underwent fixation using patient-specific plate had lower errors than those that underwent fixation using manually bending reconstruction plate and miniplate, which was the same trend as that for condylar errors, but the trend was not statistically significant.

The average condylar errors for the limit defect were smaller than those for the extensive defect in all plate types. Furthermore, manually bending reconstruction plate had significantly smaller errors $(p<0.01)$. The average fibular errors for the limit defect were smaller than those of the extensive defect for all plate types. Miniplate and manually bending reconstruction plate had significantly different errors. The overall precision of patient-specific plate was comparable in both condylar and fibular errors for all axes, and statistical analysis showed no significant difference associated with defect extent $(p>0.05)$.

Individual analysis of each plate found that the patient-specific plate had the lowest fibular and condylar errors on the x-axis (medial-lateral direction). This trend was the same for both the limit and extensive defects. The largest errors were found on the y-axis (superior-inferior direction) for all experimental models. The average errors of the patient-specific plate in both the limit and extensive defects had comparable values, which indicated the benefits of the patient-specific plate regardless of defect extent (Figure 5).

For the manually bending reconstruction plate, it was found that the fibular and condylar errors occurring in the z-axis (anterior-posterior direction) were the lowest for the limit defect. Each model in the experiment had a different twisting angle, and the errors could occur on any axis (Figures 5 and 6).

A comparison of errors according to the size of the defect found that when the defect was broad, there were significantly more errors on the $z$-axis (anterior-posterior direction) $(0.99 \pm 0.93 \mathrm{~mm}$ versus $5.30 \pm 2.70$ $\mathrm{mm}$ for the limit and extensive defect, respectively).

For miniplate fixation, the maximal condylar errors were found on the x-axis (medial-lateral direction) for both the limit and extensive defects. As a result, the mandible widened. There was no statistically significant difference in average errors according to the extent of the defect. There were higher fibular and condylar errors on the z-axis (superior-inferior direction) for extensive defect group but not statistically significant (Figure 5).

\section{Analysis of Screw Holes Position}

The locations of the screw holes were used as an indicator of the accuracy of cutting guide placement. The locations of the screw holes at the bone surface were accurate for both extent of the defect, with 
errors of less than $1 \mathrm{~mm}$ in all directions. There was no significant difference in the average error according to the extent of the defect (Figure 7).

\section{Analysis of Screw Direction}

When the screw passes through the material, it can change direction towards any axes away from the predrilled hole. For the patient-specific plate, the screw deviated from the predrilled hole to the y-axis (superior-inferior direction) in all experimental models (Figure 8). Thus, the direction of the screw, especially near the cutting surface, indicated the axis deviation of the plate. Moreover, it was found that the degree of fibular bone error was proportional to the magnitude of the screw deviation.

For the manually bending reconstruction plate, the screw also changed direction on the y-axis (superiorinferior direction), similar to the patient-specific plate, and it was found that the fibula rotated around both the $\mathrm{x}$-axis (medial-lateral direction) and y-axis (superior-inferior direction) in each bone model. The errors of the screw direction were insignificant for all axes for the miniplate.

4. Comparison of Simulation Bone Model and Reconstructed Mandible in vivo.

The errors between actual reconstructed mandible in vivo using the patient-specific plate and surgical simulation of bone model were compared. The errors were lower for the actual reconstructed mandible in vivo for all plate types and defect extents. For the limit defect, it was found that the fibular and condylar errors in the actual reconstructed mandible in vivo and surgical simulation of bone model were $1.30 \pm 0.27$ $\mathrm{mm}$ vs. $5.07 \pm 2.7 \mathrm{~mm}$ and $0.67 \pm 0.00 \mathrm{~mm}$ vs. $16.15 \pm 3.49 \mathrm{~mm}$, respectively (Figure 9).

For the extensive defect, it was found that the fibular and condylar errors for the actual reconstructed mandible in vivo and the surgical simulation of bone models were $4.78 \pm 1.14 \mathrm{~mm}$ vs. $5.07 \pm 2.7$ and $1.8 \pm 0.0 \mathrm{~mm}$ vs. $8.09 \pm 2.52$, respectively.

The fibular errors for the surgical simulation of bone models and the actual reconstructed mandible in vivo were higher than the condylar errors for both extent of defects but were not significant. The average condylar errors of the actual reconstructed mandible in vivo were lower than that in the surgical simulation of bone models, and this finding occurred for all axes of measurement. Furthermore, the errors that were found frequently in the bone model on the y-axis (superior-inferior direction) did not have a high value in the actual reconstructed mandible in vivo.

\section{Discussion}

CAS in mandibular reconstruction with fibular free flaps has become a new standard in medical treatment. A recent systematic review and meta-analysis showed various benefits, such as increased precision and accuracy in reconstruction, decreased operative time ${ }^{6,13}$ and increased long-term stability 14. 
In general, it was found that CAS for fibular free flaps can be divided into two parts. The first part is for surgical planning and creating cutting guides to assist in the surgery. The second part involves mandibular plate preparation for the fixation. According to published literatures, the subjects were separated into three categories by using the different plate fixation methods. The first group used CAD/CAM combined with patient-specific plate ${ }^{14-16}$. The second group used CAD/CAM combined with manually bending reconstruction plate ${ }^{7,8,17-25}$. The last group used CAD/CAM combined with miniplate $26,27$.

The factors affecting the selection of fixation plate are the availability of equipment, urgency of the operation, surgical planning preparation time, 3D printing process, CAD/CAM equipment transportation time, and expenses. Moreover, CAD/CAM technology has limited availability, only special laboratories in some hospitals have this service. So, the patient-specific plate are not always available for use in mandibular reconstruction. For the alternative ways of the aforementioned issue, the authors aimed to determine which plate fixation technique is suitable and assists reconstruction of mandible with fibular free flaps.

\section{Patient-specific Plate}

Regarding patient-specific plate, we hypothesized that the cutting guide determines the accuracy of the mandibular reconstruction by affecting the positioning of screws. The condylar errors were greatest on the $y$-axis (superior-inferior direction). After we analysed the position of the screws on the bone surface, it was found that there was only a petite error $(0.56 \pm 0.38 \mathrm{~mm})$ that was not proportionate to the condylar error $(8.09 \pm 2.52 \mathrm{~mm})$, which was 14 times greater. Therefore, an in-depth analysis of screws direction was conducted. Apart from screw position at the bone surface, the errors of screw direction inside the bone were high on the y-axis (superior-inferior direction) and directly resulted in the condylar error. The average errors of screw hole position at the bone surface were not significantly different regarding extent of the defect, which indicated the accuracy of the screw hole direction at the point of entrance. The screws that located near osteotomy sites affected the condylar errors for both direction and magnitude. Screw direction shifting was also found with the manually bending reconstruction plate. The underlying cause of direction shift in manually bending reconstruction plate was suspected from the relatively large screw size, which is more resistant the bone, resulting in a deviation of the screw from the predrilled axis. The finding supporting this hypothesis was that no significant error was found for fixation with miniplate, which uses smaller screws. In contrast, the smallest error on the x-axis (medial-lateral direction) could be explained by the supporting forces from surrounding soft tissue and TMJ on both sides of mandibular condyle.

Therefore, to reduce error on the $y$-axis (superior-inferior direction), the cutting guide must be meticulously assembled and applied, and the screws must be installed precisely in all axes through the predrilled tract.

According to the results of the current study, patient-specific plate do not have significant benefits over manually bending reconstruction plate in terms of accuracy in the limit defect. This may be due to the 
ability of surgeons to bend the commercially available manually bending reconstruction plate for short and uncomplicated defects using the hands and eyes. However, the surgeons' hand skills cannot overcome the challenge of extensive defects in the mandible, as we will describe in the next section.

\section{Manually Bending Reconstruction Plate}

The error of the manually bending reconstruction plate was highest on the z-axis (antero-posterior direction). We believe that this error occurred from the difficulty of plate bending, which was impeded by plate thickness. Surgeons can use the bone model of mandible or the native mandible as a reference. The main bending points are usually the points that can be clearly seen, such as the seam between the anterior and lateral part of the mandible, by bending the plate at significant points, which normally have 1-3 angles. However, in reality, the mandible is gradually curving and changes direction for two or more axes along its part. The limitation of manually bending resulted in the inadequate alignment between plate and bone surface. Furthermore, tipping and ejection of plate on another side was occurred after fixation of plate on one side. The shifting of plate in antero-posterior axis (z-axis) might be occurred from fixation force that try to align the plate on mandible and there is room for the plate in this direction only (Figure 10).

The patient-specific plate has not only a great accuracy but also the durability to force load. There was an in vitro study by Kasper et al., which reported that patient-specific plate were seem to benefit over manually bending reconstruction plate in terms of durability against physiological force loading ${ }^{16}$. These results suggested the benefit of using patient-specific plate over manually bending reconstruction plate.

\section{Miniplate}

Most of published literatures focused on stability of fixation and postoperative complications between using miniplate and manually bending reconstruction plate. The difference in complications between these two plates remained controversial ${ }^{28-30}$. A finite element study showed better stability in manually bending reconstruction plate over miniplate ${ }^{31}$. Despite this question was critical, the data regarding the accuracy of miniplate fixation were limited.

In our recent study, it was found that using of miniplate could create condylar error on any axis. The leading cause of this error might occur from the uncontrollable overall shape of neo-mandible upon miniplate fixation. The aforementioned error was solved by using a "bar" to connect between both sides of the cutting guide resulted in regulation of shape of the fibular segment and miniplate complex ${ }^{32}$. Nevertheless, there were factors involving errors of fixation, not only the bone, cutting guide, and plate complex, but also the forces from surrounding soft tissues, which distorted the plate and neo-mandible system postoperatively. Up to the present date, the studies on long-term outcomes of miniplate fixation are limited. Therefore, as the current evidence suggests, we do not recommend miniplate fixation as the first place. The summary of the errors in each plate type and recommendations is given in Table 1. 
Table 1

The errors in each plate type and recommendations. The miniplate, manually bending reconstruction plate, and patient-specific plate had their own errors and limitations. However, there are several ways to

minimize these errors, and surgeons should consider these issues to achieve favourable outcomes.

\begin{tabular}{|c|c|c|}
\hline Type of plate & Findings & Management \\
\hline Miniplate & $\begin{array}{l}\text { - Errors can occur in any direction. } \\
\text { - The greatest error occurs along the } \\
\text { x-axis. }\end{array}$ & $\begin{array}{l}\text { - Use other types of plate instead of a } \\
\text { miniplate. } \\
\text { - Use connecting bar with a cutting guide } \\
32 \text {. } \\
\text { - Avoid widening gap between the fibula } \\
\text { and plate lining. }\end{array}$ \\
\hline $\begin{array}{l}\text { Manually } \\
\text { bending } \\
\text { reconstruction } \\
\text { plate }\end{array}$ & $\begin{array}{l}\text { - The manually bending } \\
\text { reconstruction plate is not fit to the } \\
\text { mandible. } \\
\text { - Increasing error in extensive } \\
\text { defects. } \\
\text { - Errors of screw direction occur } \\
\text { beyond the bone surface. }\end{array}$ & $\begin{array}{l}\text { - Avoid using manually bending } \\
\text { reconstruction plate for extensive defect } \\
\text { reconstruction. } \\
\text { - Avoid forceful drilling in patients with } \\
\text { osteoporosis. } \\
\text { - Direction of screws should follow the } \\
\text { predrilled axis. } \\
\text { - The screw should be gently inserted } \\
\text { along the predrilled tract to avoid false } \\
\text { tract creation. }\end{array}$ \\
\hline $\begin{array}{l}\text { Patient-specific } \\
\text { plate }\end{array}$ & $\begin{array}{l}\text { Errors of screw direction occur } \\
\text { beyond the bone surface. (especially } \\
\text { on the z-axis). }\end{array}$ & $\begin{array}{l}\text { - Direction of screws should follow the } \\
\text { predrilled axis. } \\
\text { - Avoid forceful drilling in patients with } \\
\text { osteoporosis. } \\
\text { - The screw should be inserted gently } \\
\text { along the predrilled tract to avoid false } \\
\text { tract creation. }\end{array}$ \\
\hline
\end{tabular}

\section{Clinical Case Analysis}

From the analysis of errors in the actual reconstructed mandible in vivo, there was lesser errors than the surgical simulation in bone model. Several hypotheses had been proposed for this manifestation. First, the bone model has a different internal supporting structure from human bone, which might cause different errors. In this regard, the surgeon may need to avoid forceful drilling when using relatively large screws, which are compatible with some types of plate, in patients who are fragile and/or have low bone density, such as elderly patients and those with osteoporosis. Furthermore, screws should be gently inserted along the predrilled axis to avoid false tract creation. Second, as mentioned above, is that in the actual patient, the surrounding soft tissues and both TMJs serve as support structures and guides for plate position in a functional position. 
For the comparison of errors, the fibular error was greater than the mandibular error since the two errors in fixation occur first with the mandibular screw and second with the fibular screw. Hence, screw application and plate fixation should be performed meticulously to reduce this error.

Gravvanis et al. ${ }^{33}$ recommended reattaching the masseter muscle to the neo-mandible to help reduce the patient's recovery time. On the other hand, this small error may not affect the patient's functional outcome, and one could question the value of the investment, in terms of cost and time, in CAD/CAM and patient-specific plate. For example, the issue of minimal clinically important differences (MCIDs) in mandibular reconstruction remains a question that requires further study 12 .

\section{Limitations Of The Study}

In this study, a few limitations should be considered when interpreting the experimental results. First, the sample size was relatively small among the published literatures ${ }^{16,26,27}$. Second, there were differences between the surgical simulation in bone models and actual reconstructed mandible in vivo, such as the screw direction errors, as detected in this study. In this case, we needed to consider the potential errors in the bone model itself. Furthermore, we should reconsider the interpretation of the results of previous studies conducted in models as well as those in future studies.

\section{Conclusions}

Despite using CAS combined with a cutting guide, errors still occurred, though they varied among plate fixation types. However, CAS combined with patient-specific plate had significantly smaller errors for both extents of the defect. Furthermore, this study showed that CAD/CAM in combination with patient-specific plate has benefits over other types of plate fixation in extensive defects as it reduces the condylar error, which other plate fixation methods cannot achieve. Interestingly, the errors of the patient-specific plate from the actual reconstructed mandible in vivo were lower in terms of both fibular and condylar errors, which might occur from the compensation forces of surrounding structures. Each plate type had limitations and errors, and surgeons should be aware of these issues. In conclusion, this study might support and ignite the further clinical studies on the accuracy of mandible reconstruction in various aspects.

\section{Declarations}

\section{Acknowledgements}

Thanks to Nachasa Khongchu from the Research Department, Faculty of Medicine Siriraj Hospital, Mahidol University for data analysis and Chanya Sinmaroeng from the Faculty of Medicine Siriraj Hospital, Mahidol University for data enrolment and technical issues.

\section{Author contributions}


NY: Project initiation \& development, data analysis, manuscript writing and editing

KR and NV : data collection and data analysis

IC and JT : data correction and analysis

NK : data analysis, manuscript writing and editing

MO and PP : Manuscript editing and data analysis

CP: Project initiation \& development, data analysis

All authors reviewed the manuscript.

\section{Competing Interests Statement}

The authors declare no competing interests.

\section{Data availability}

The datasets used and/or analysed during the current study available from the corresponding author on reasonable request.

\section{References}

1. Hemmy, D. C., David, D. J. \& Herman, G. T. Three-dimensional reconstruction of craniofacial deformity using computed tomography. Neurosurgery 13, 534-541, doi:10.1227/00006123-198311000-00009 (1983).

2. Vannier, M. W., Marsh, J. L. \& Warren, J. O. Three dimensional CT reconstruction images for craniofacial surgical planning and evaluation. Radiology 150, 179-184, doi:10.1148/radiology.150.1.6689758 (1984).

3. Ueda, K. et al. Mandibular reconstruction using computer-generated three-dimensional solid models. J Reconstr Microsurg 10, 291-296; discussion 296-297, doi:10.1055/s-2007-1006597 (1994).

4. Hanasono, M. M. \& Skoracki, R. J. Computer-assisted design and rapid prototype modeling in microvascular mandible reconstruction. Laryngoscope 123, 597-604, doi:10.1002/lary.23717 (2013).

5. Ciocca, L. et al. CAD/CAM guided secondary mandibular reconstruction of a discontinuity defect after ablative cancer surgery. J Craniomaxillofac Surg 40, e511-515, doi:10.1016/j.jcms.2012.03.015 (2012).

6. Rodby, K. A. et al. Advances in oncologic head and neck reconstruction: systematic review and future considerations of virtual surgical planning and computer aided design/computer aided modeling. $J$ Plast Reconstr Aesthet Surg 67, 1171-1185, doi:10.1016/j.bjps.2014.04.038 (2014). 
7. Roser, S. M. et al. The accuracy of virtual surgical planning in free fibula mandibular reconstruction: comparison of planned and final results. J Oral Maxillofac Surg 68, 2824-2832, doi:10.1016/j.joms.2010.06.177 (2010).

8. Lethaus, B. et al. Additive manufacturing for microvascular reconstruction of the mandible in 20 patients. J Craniomaxillofac Surg 40, 43-46, doi:10.1016/j.jcms.2011.01.007 (2012).

9. Zavattero, E. et al. Accuracy of Fibula Reconstruction Using Patient-Specific Cad/Cam Plates: A Multicenter Study on 47 Patients. Laryngoscope 131, E2169-E2175, doi:10.1002/lary.29379 (2021).

10. Wang, L., Liu, K., Shao, Z. \& Shang, Z. J. Management of the condyle following the resection of tumours of the mandible. Int J Oral Maxillofac Surg 46, 1252-1256, doi:10.1016/j.ijom.2017.04.029 (2017).

11. Nahabedian, M. Y., Tufaro, A. \& Manson, P. N. Improved mandible function after hemimandibulectomy, condylar head preservation, and vascularized fibular reconstruction. Ann Plast Surg 46, 506-510, doi:10.1097/00000637-200105000-00009 (2001).

12. Yang, W. F. et al. Spatial deviations of the temporomandibular joint after oncological mandibular reconstruction. Int J Oral Maxillofac Surg, doi:10.1016/j.ijom.2021.02.033 (2021).

13. Brown, J. S., Lowe, D., Kanatas, A. \& Schache, A. Mandibular reconstruction with vascularised bone flaps: a systematic review over 25 years. Br J Oral Maxillofac Surg 55, 113-126, doi:10.1016/j.bjoms.2016.12.010 (2017).

14. Zeller, A. N. et al. Patient-Specific Mandibular Reconstruction Plates Increase Accuracy and LongTerm Stability in Immediate Alloplastic Reconstruction of Segmental Mandibular Defects. $J$ Maxillofac Oral Surg 19, 609-615, doi:10.1007/s12663-019-01323-9 (2020).

15. Sweed, A. H. et al. Factors influencing CAD/CAM accuracy in fibula free flap mandibular reconstruction. Acta Otorhinolaryngol Ital 40, 138-143, doi:10.14639/0392-100X-N0400 (2020).

16. Kasper, R., Winter, K., Pietzka, S., Schramm, A. \& Wilde, F. Biomechanical In Vitro Study on the Stability of Patient-Specific CAD/CAM Mandibular Reconstruction Plates: A Comparison Between Selective Laser Melted, Milled, and Hand-Bent Plates. Craniomaxillofac Trauma Reconstr 14, 135143, doi:10.1177/1943387520952684 (2021).

17. Ueda, K. et al. Mandibular contour reconstruction with three-dimensional computer-assisted models. Ann Plast Surg 46, 387-393, doi:10.1097/00000637-200104000-00006 (2001).

18. Derand, P. \& Hirsch, J. M. Virtual bending of mandibular reconstruction plates using a computeraided design. J Oral Maxillofac Surg 67, 1640-1643, doi:10.1016/j.joms.2009.03.039 (2009).

19. Sharaf, B. et al. Importance of computer-aided design and manufacturing technology in the multidisciplinary approach to head and neck reconstruction. J Craniofac Surg 21, 1277-1280, doi:10.1097/SCS.0b013e3181e1b5d8 (2010).

20. Antony, A. K., Chen, W. F., Kolokythas, A., Weimer, K. A. \& Cohen, M. N. Use of virtual surgery and stereolithography-guided osteotomy for mandibular reconstruction with the free fibula. Plast Reconstr Surg 128, 1080-1084, doi:10.1097/PRS.0b013e31822b6723 (2011). 
21. Bell, R. B., Weimer, K. A., Dierks, E. J., Buehler, M. \& Lubek, J. E. Computer planning and intraoperative navigation for palatomaxillary and mandibular reconstruction with fibular free flaps. $J$ Oral Maxillofac Surg 69, 724-732, doi:10.1016/j.joms.2009.12.040 (2011).

22. Zheng, G. S. et al. Mandible reconstruction assisted by preoperative virtual surgical simulation. Oral Surg Oral Med Oral Pathol Oral Radiol 113, 604-611, doi:10.1016/j.tripleo.2011.05.016 (2012).

23. Shen, Y. et al. Using computer simulation and stereomodel for accurate mandibular reconstruction with vascularized iliac crest flap. Oral Surg Oral Med Oral Pathol Oral Radiol 114, 175-182, doi:10.1016/j.tripleo.2011.06.030 (2012).

24. Naros, A. et al. Three-dimensional accuracy of mandibular reconstruction by patient-specific pre-bent reconstruction plates using an "in-house" 3D-printer. J Craniomaxillofac Surg 46, 1645-1651, doi:10.1016/j.jcms.2018.05.047 (2018).

25. Goormans, F. et al. Accuracy of computer-assisted mandibular reconstructions with free fibula flap: Results of a single-center series. Oral Oncol 97, 69-75, doi:10.1016/j.oraloncology.2019.07.022 (2019).

26. Steffen, C. et al. Biomechanical comparison of polylactide-based versus titanium miniplates in mandible reconstruction in vitro. J Stomatol Oral Maxillofac Surg 121, 377-382, doi:10.1016/j.jormas.2019.12.001 (2020).

27. Steffen, C. et al. Biomechanical comparison of titanium miniplates versus a variety of CAD/CAM plates in mandibular reconstruction. J Mech Behav Biomed Mater 111, 104007, doi:10.1016/j.jmbbm.2020.104007 (2020).

28. Al-Bustani, S. et al. Miniplates Versus Reconstruction Bars for Oncologic Free Fibula Flap Mandible Reconstruction. Ann Plast Surg 77, 314-317, doi:10.1097/SAP.0000000000000497 (2016).

29. Robey, A. B. et al. Comparison of miniplates and reconstruction plates in fibular flap reconstruction of the mandible. Plast Reconstr Surg 122, 1733-1738, doi:10.1097/PRS.0b013e31818a9ac5 (2008).

30. Zhang, Z. L., Wang, S., Sun, C. F. \& Xu, Z. F. Miniplates Versus Reconstruction Plates in Vascularized Osteocutaneous Flap Reconstruction of the Mandible. J Craniofac Surg 30, e119-e125, doi:10.1097/SCS.0000000000005020 (2019).

31. Park, S. M., Lee, J. W. \& Noh, G. Which plate results in better stability after segmental mandibular resection and fibula free flap reconstruction? Biomechanical analysis. Oral Surg Oral Med Oral Pathol Oral Radiol 126, 380-389, doi:10.1016/j.000o.2018.05.048 (2018).

32. Lim, S. H., Kim, M. K. \& Kang, S. H. Precision of fibula positioning guide in mandibular reconstruction with a fibula graft. Head Face Med 12, 7, doi:10.1186/s13005-016-0104-2 (2016).

33. Gravvanis, A., Anterriotis, D. \& Kakagia, D. Mandibular Condyle Reconstruction With Fibula FreeTissue Transfer: The Role of the Masseter Muscle. J Craniofac Surg 28, 1955-1959, doi:10.1097/SCS.0000000000003998 (2017).

\section{Figures}


$1 \mathrm{a}$

\section{Limit defect}

The boundarv started from the right nara-svmnhvsis

\section{Figure 1}

Types of mandibular defects. The defect that involved the para-symphysis and ipsilateral angle of mandible was defined as a "limit defect" (figure 1a), and the defect that involved the body of the mandible to the contralateral angle of the mandible was defined as an "extensive defect" (figure 1b). 


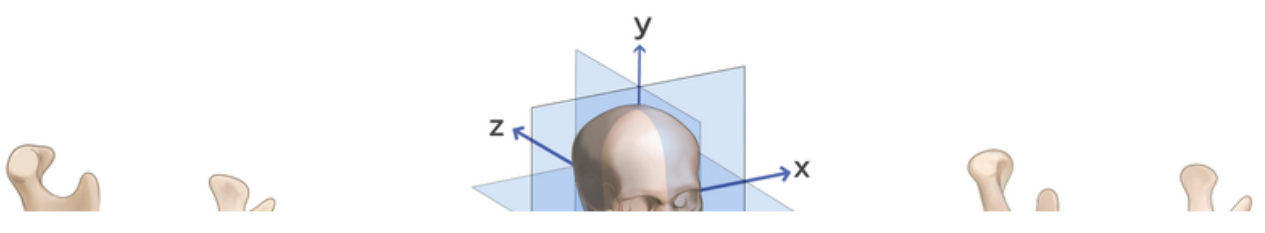

\section{Figure 2}

The allocation of bone model experiments among defect types and mandibular plates. Each type of defect was fixed with different types of plate, including miniplate, bendable reconstruction plate, and patient-specific plate. All models were fixed with each type of plate three times, and the results were averaged. 

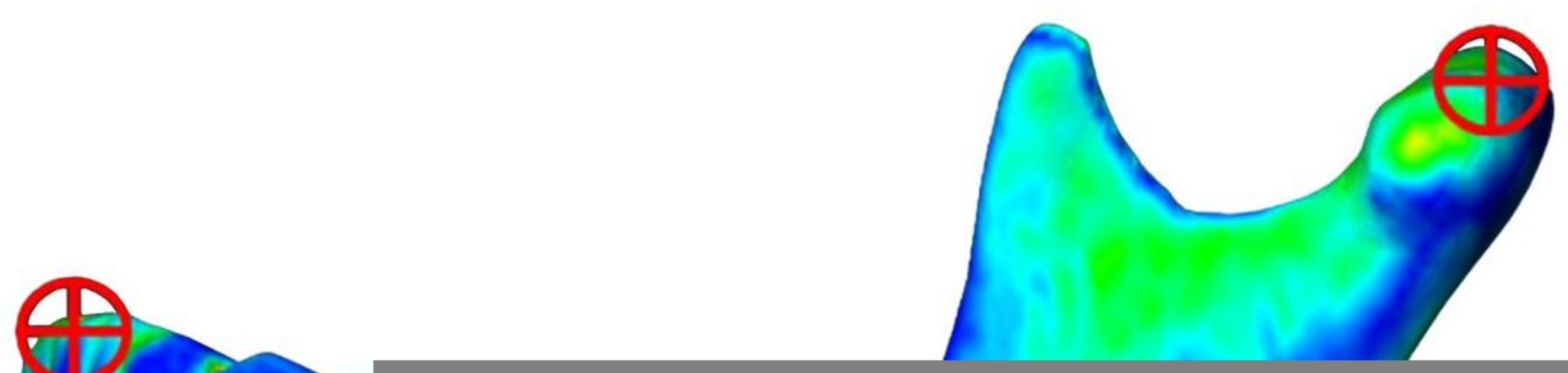

\section{Figure 3}

The superimposed image containing the CT scanning images of the reconstructed model and the preoperative CT scanning images of the mandible. The five main reference points on the mandible are shown in red-crossed circles, and each point had to have a gap of less than $1 \mathrm{~mm}$ between the preoperative and reconstructed images. For the colour gradient on the mandible surface, which represents differences between models, red indicates the greatest difference, while green represents a smaller difference. 


\begin{tabular}{|c|c|}
\hline $4 a$ & Condylar error (Limit defect) \\
\hline 35 & Patient-specific plate \\
\hline
\end{tabular}

$4 b$

20
Fibular error (Limit defect)

@ Patient-specific plate

$=$ MAanualls, handina rarnnctrurtinn nlato

\section{Figure 4}

Three-dimensional analysis of errors by plate type and defect extent. The errors in the 3D axes $(x-, y-$, and $z$-axis) were analysed separately and on average. The average error between the fibula and condyle was not significantly different among plate types for the limit defect (figure 4a-b). However, there was significantly low condylar error with patient-specific plate in the extensive defect group $(p<0.05)$ and less fibular error, but the difference was not statistically significant (figure 4c-d). 

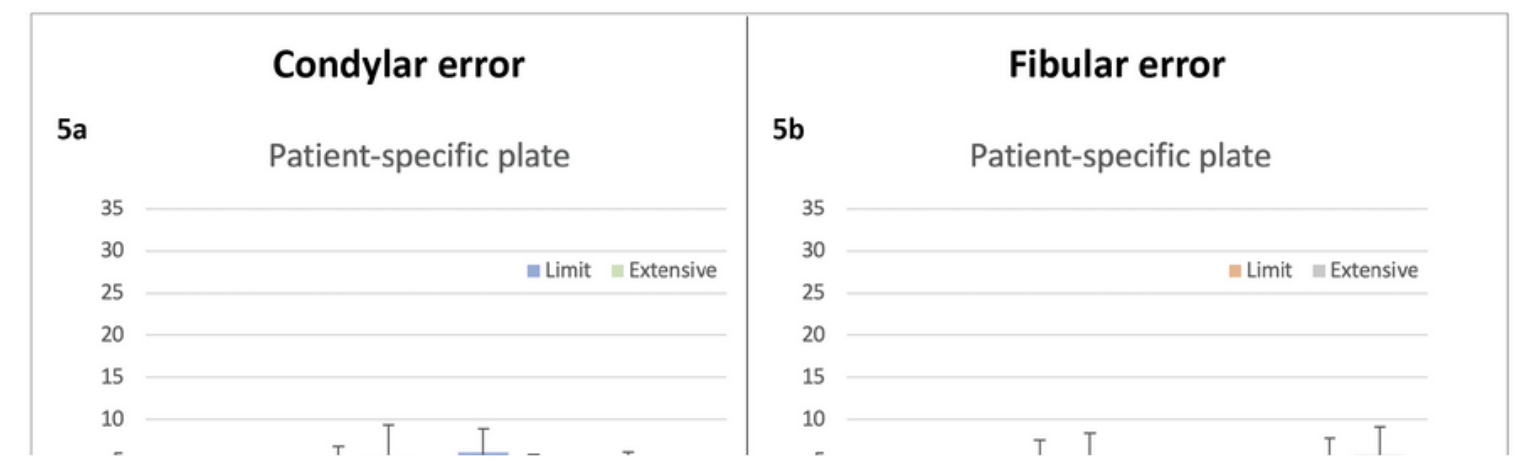

\section{Figure 5}

Comparison of error between defect extent groups by plate type. The patient-specific plate had comparable condylar and fibular errors regardless the defect extent. The extent of the mandible defect had less effect on patient-specific plate error (figure 5a-b). The manually bending reconstruction plate can have an error for any axis, and there was a significant difference in average errors between the limit and extensive defect groups. (figure $\mathbf{5 c - d}$ ). For miniplate, the average errors were generally higher than those 
for other plate types, and the average errors were higher in extensive defect group but not statistically significant (figure $5 \mathbf{e} f$ ).

\section{Figure 6}

The three-dimensional (3D) analysis of the manually bending reconstruction plate and mandible. The errors of manually bending reconstruction plate could occur on any axis (figure 6a-b).

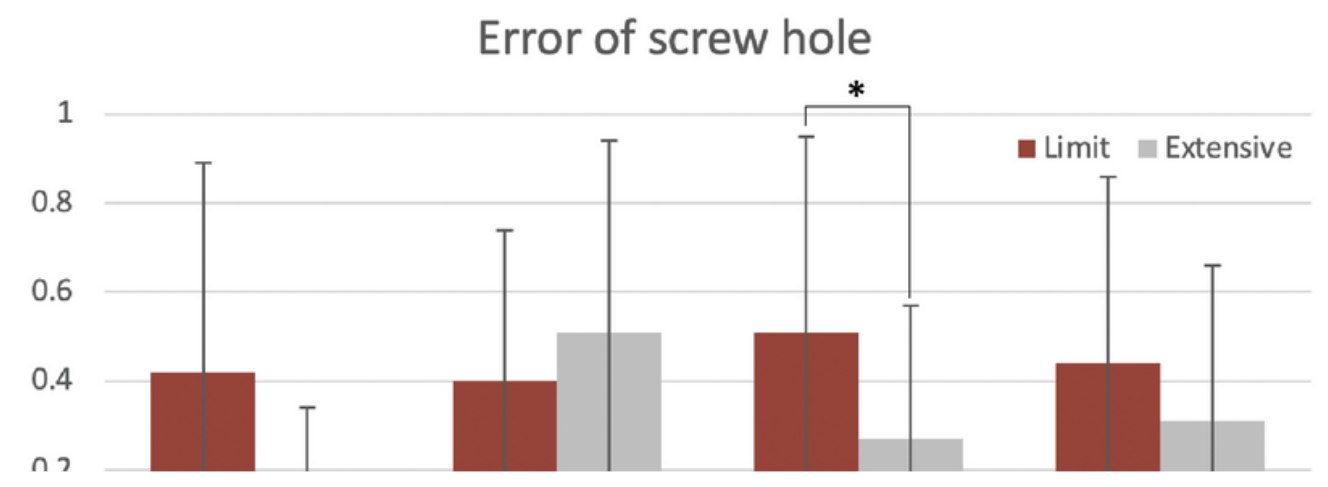




\section{Figure 7}

The errors of screw hole position at the bone surface. The errors of screw holes were significantly different between limit and extensive defect in the $x$ and $z$-axis $(p<0.05)$. However, the average errors in all axes were not significantl y different.
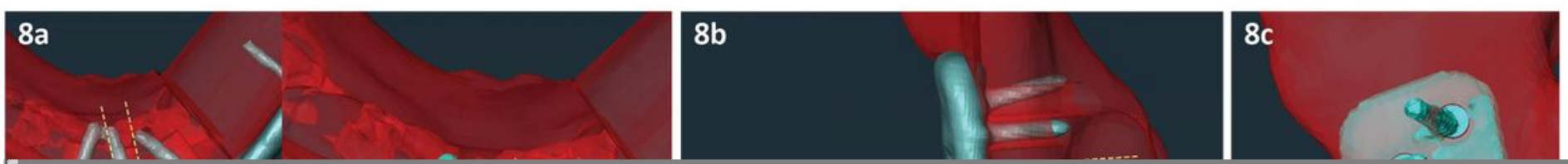

\section{Figure 8}

3D analysis of screw direction in the mandibular model. The direction of the screws deviated from the predrilled hole, and this deviation could occur on any axis (figure 8a-c). For the patient-specific plate, the screw direction deviated from the predrilled hole towards the $y$-axis for both extent of the defect (figure

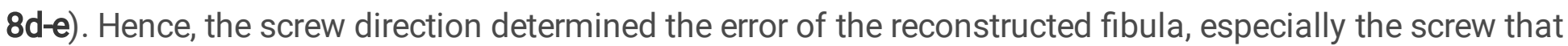
located near the osteotomy site.

\section{Figure 9}

Comparison of the errors of patient-specific plate in actual reconstructed mandible in vivo and surgical simulation of bone models. The average condylar and fibular errors in limit defect of the actual reconstructed mandible in vivo were lower than surgical simulation of bone models (figure $9 a-b)$. For the 
extensive defect, the average condylar and fibular errors were also lower in the actual reconstructed mandible in. (figure $9 \mathbf{c - d}$ ). The condylar error of the actual reconstructed mandible in vivo had a lower error than the bone model for all axes. Interestingly, the y-axis error diminished in the actual reconstructed mandible in vivo for both extent of defects.

\section{$10 a$}


The mechanism of errors in manually bending reconstruction plate. The manually bending reconstruction plate was difficult to bend. The bending points were related to mandible shape, and the plate was bent with only 1-3 angles, which were not meticulously enough to fit the alignment of mandible curvature and direction (figure 10a). In addition, the plate was further displaced in antero-posterior (z-axis) direction by the fixation force that try to align the plate with the mandible (figure 10b). 